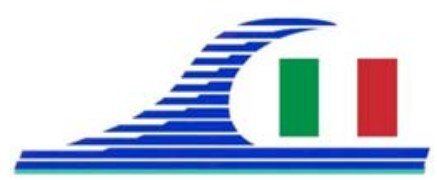

Conférence Méditerranéenne Côtière et Maritime EDITION 3, FERRARA, ITALIA (2015)

Coastal and Maritime Mediterranean Conference

Disponible en ligne - http://www.paralia.fr - Available online

\title{
Degré de pollution marine par les métaux lourds dans les sédiments superficiels des principaux ports algériens
}

\author{
Farid ATROUNE ${ }^{1}$
}

1. Laboratoire de Géo-Environnement, Faculté des Sciences de la Terre, de Géographie et de l'aménagement du Territoire USTHB BP 32, Bab Ezzouar El Alia 16111, Alger Algérie.faridatroune@gmail.com

\section{Résumé :}

La distribution des métaux lourds dans les sédiments marins des ports algériens est régie par plusieurs facteurs: la granulométrie, la teneur en matière organiques et la localisation des sources de pollution.

La fraction fine du sédiment piège un nombre important de métaux lourds; résultat probable de l'importante activité au niveau des ports et à l'exposition directe aux effluents d'origine tellurique. Elle dépend des conditions hydrodynamiques et hydrosédimentaires qui régissent les baies (Oran, Alger et Annaba) et traduit aussi les influences anthropiques.

Les quantités et la composition de la matière organique dissoute, colloïdale et particulaire joue un rôle important dans les transferts des contaminants ainsi que leur transformation (SCHWARZENBACH et al., 2003). Le phénomène de floculation (CHESTER \& STONER, 1985) et de décantation se charge de la distribution de ces dépôts dans ces différents ports.

La comparaison du degré de contamination des métaux lourds des sédiments superficiels des 3 ports choisies montrent clairement que le port de Annaba est le plus pollué puis le port d'Alger et en fin le port d'Oran.

Mots clés : Granulométrie, Métaux lourds, Pollution, Ports, Alger, Oran et Annaba.

\section{Introduction}

En méditerranée les sédiments résultant d'une production allochtone ou autochtone du milieu comblent les ports, les estuaires et les cours d'eau. Ces sédiments entravent la circulation des navires qui traversent chaque jour les sites portuaires. Des opérations de dragage ont toujours été indispensables afin d'entretenir, approfondir ou aménager ces sites et assurer le bon fonctionnement du commerce par voies maritimes et fluviales. Néanmoins, des substances polluantes sont le plus souvent piégées dans ces sédiments. La mise en dépôt terrestre et le rejet en mer étaient les seules solutions de gestion de ces matériaux indésirables (RAMADE, 1991).

L'urbanisation non contrôlée des bassins et la présence de sites industriels à risque élevé, proche des concentrations urbaines, ont générées des pollutions et des 
Côtes méditerranéennes menacées :

Risques et défis dans le contexte du changement climatique

dégradations. Les métaux lourds piégés dans les sédiments superficiels des ports algériens en sont la preuve (ATROUNE \& BOUTALEB, 2012).

Les métaux lourds sont toujours associés à la fraction fine (ROBBE, 1981). Les différents oueds se jetant dans la baie d'Oran, d'Alger et d'Annaba drainent divers types de pollution.

\section{Caractéristiques de la zone d'étude}

Le port Oran est situé au fond d'une baie de 16 milles de large sur 7 milles de profondeur ouverte aux vents dominants et aux grosses tempêtes de Nord-Nord-Ouest, limitée à l'Ouest par le Cap Falcon et à l'Est par la pointe de l'Aiguille ,celui d'Annaba est situé dans la partie Sud-Ouest d'un golfe. La chaîne de l'Edough, orientée Sud-Ouest à Nord-Est et terminée par un promontoire abrupt, le Cap de Garde, le met en effet à l'abri des vents dominants du Nord-Ouest et d'Ouest.Le port d'Alger est situé dans la partie Ouest qui se présente en forme d'un croissant de 10 milles environ de cordé et de 4 milles de flèche, largement ouvert sur le Nord et dont les pointes sont, à l'Ouest, le cap Caxine et à l'Est le Cap Matifou (Tamentfoust), (Fig.1).

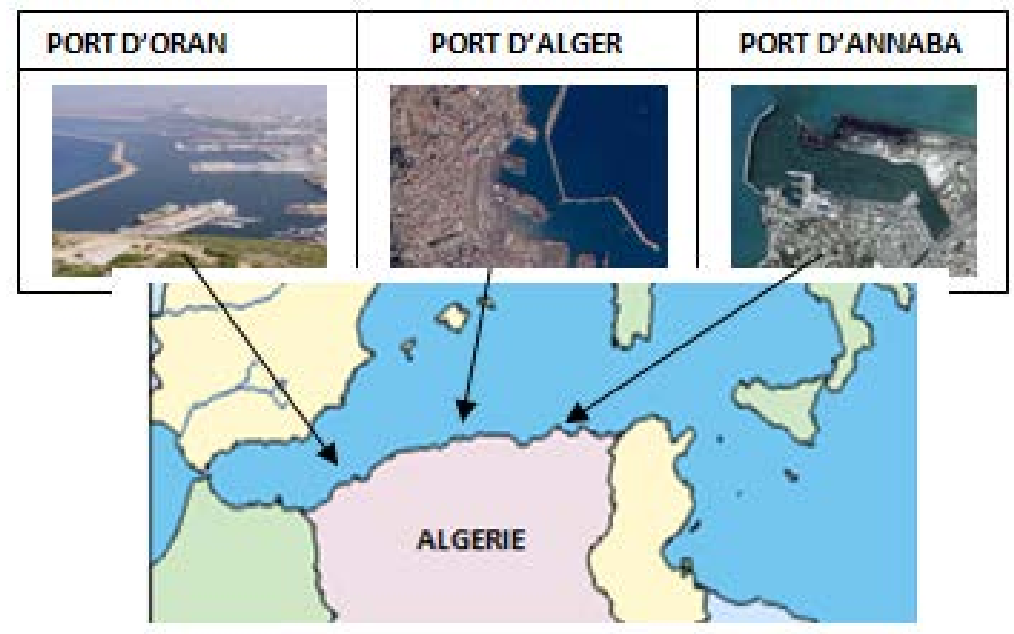

Figure 1. Situation géographique des 3 zones d'études.

\section{Matériels et méthodes d'étude}

Le nombre d'échantillons des sédiments de surface a été réalisé à des périodes différentes (40 en mai 2009 au port d'Oran, 52 au port d'Alger en juin 2010 et 47 en mai 2011 au port d'Annaba,). Seuls 20 échantillons ont été choisis d'une manière ponctuelle dans chaque port au milieu des darses en fonction de la granulométrie. Les échantillons sont prélevés à l'aide d'une benne Van Veen à des profondeurs allant de 8 à $15 \mathrm{~m}$; seule la couche superficielle, crème de vase (environ $0.5 \mathrm{~cm}$ ) est conservée pour l'analyse des métaux lourds (poids sec). Le reste du sédiment est destiné à la granulométrie de la fraction fine. 
Mediterranean coasts at threat:

Hazards and challenges in the context of climate variability

Les dosages ont été réalisés à l'aide d'un spectrophotomètre d'absorption atomique à flamme (Shimadzu AA-6601). La concentration des éléments métalliques est obtenue par la formule de JOANNY et al. (1980).

\section{Résultats et discussion}

Du point de vue granulométrie dans la zone de protection des ports, apparaissent des mélanges de facies de types sableux et de vases sableux traduisant une zone à régime hydrodynamique faible. Les courbes granulométriques de la fraction inférieure à 40 microns sont généralement unimodales à allure hyperbolique. Seules les proportions respectives (indices d'évolution, médianes, ...) changent.

Les échantillons analysés aux niveaux des différentes darses d'Annaba d'Oran et d'Alger concernent des résultats ponctuels (maxima) en poids sec. Elles montrent des taux élevés en ETM (Tab 1, Fig. 2). En effet les résultats ont permis de mettre en évidence une association de trois éléments le $\mathrm{Zn}, \mathrm{Pb}$ et $\mathrm{Cu}$ qui représentent un taux de contamination important, ce qui rejoint les résultats obtenus par les travaux de BELABED (2010) au port d'Annaba et les travaux d'ATROUNE \& BOUTALEB (2012) au port d'Alger. La valeur obtenue au port d'Annaba reste la plus remarquable, 6 fois la valeur guide (ONEDD \& JICA, 2007) pour le cuivre, 12 fois celle du plomb et 10 fois celle du mercure.

Tableau 1. Comparaison des différentes concentrations en métaux lourds des 3 ports (Oran, Alger et Annaba) algériens.

\begin{tabular}{llllllllll}
\hline $\begin{array}{l}\text { Métaux lourds } \\
(\mu \mathrm{g} / \mathrm{F})\end{array}$ & $\mathrm{Fe}$ & $\mathrm{Cu}$ & $\mathrm{Zn}$ & $\mathrm{Cr}$ & $\mathrm{Mn}$ & $\mathrm{Ni}$ & $\mathrm{Pb}$ & $\mathrm{Cd}$ & $\mathrm{Hg}$ \\
\hline Port d'Oran & - & 60.70 & 321 & - & - & 35 & 87 & 0.21 & 0.84 \\
Port D'Alger & 18.84 & 90.21 & 356 & - & 170.35 & - & 186 & 1.20 & 1.28 \\
Port d'Annaba & 19,75 & 140.7 & 643 & 151 & 42,5 & 47 & 290 & 3.8 & 2.04 \\
Valeurs Guide & 2000 & 26 & 88 & 400 & 45 & 45 & 22 & 0.6 & 0.2 \\
\hline
\end{tabular}
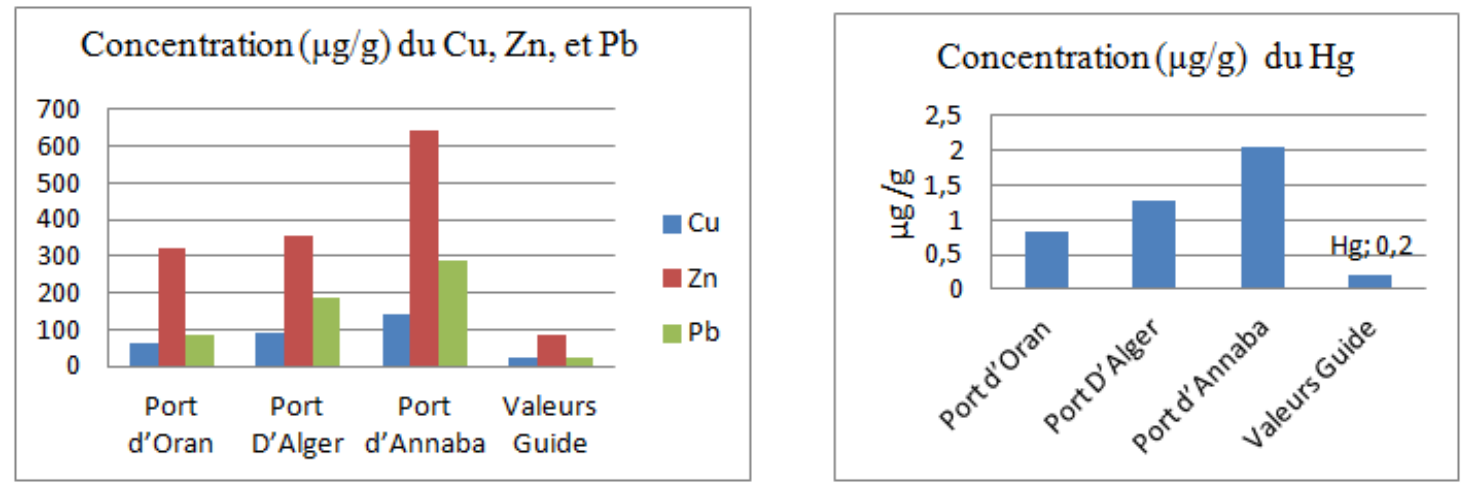

Figure 2. Distribution des concentrations de $\mathrm{Cu}, \mathrm{Zn}, \mathrm{Pb}$ et $\mathrm{Hg}$ dans les sédiments superficiels des ports d'Oran, Alger et d'Annaba. 
Côtes méditerranéennes menacées :

Risques et défis dans le contexte du changement climatique

\section{Conclusions}

Les fortes variations des teneurs en métaux lourds témoignent de l'existence de plusieurs sources de pollution :

- Rejets des affluents domestiques et industriels dans les ports et les baies respectifs par les oueds.

- A la dispersion du panache des eaux usées déversés dans la mer a la canalisation combinée du transit littoral.

Les résultats ont montré une association de métaux lourds constitués par le $\mathrm{Pb}$, $\mathrm{Cu}$ et $\mathrm{Zn}$ et le mercure Hg qui dépasse dangereusement le minimum requis.

Vue l'état de pollution critique des sédiments superficiels par les métaux lourds, la nécessité d'implantation de stations d'épuration des eaux usées s'avère d'une importance majeure pour améliorer la qualité des eaux et l'environnement marin côtier et portuaire.

\section{Références bibliographiques}

ATROUNE F., BOUTALEB A. (2012). Les métaux lourds dans les sédiments superficiels de la baie d'Alger : Influence de l'Oued El Harrach sur leur distribution. Bulletin du service géologique National. Vol. 23(2), pp 169-178.

BELABED B.E. (2010). La pollution par les métaux lourds dans la région de Annaba "sources de contamination des écosystèmes aquatiques". Thèse de doctorat. Université Badji Mokhtar de Annaba. 180 p.

CHESTER R., STONER J.M. (1985). Trace elements in total particulate material from surface sea-water. Nature 255, pp 50-51.

JOANNY M., CHAUSSEPIED M., CORRE F. (1980). Présentation des résultats d'une calibration internationale. Centre national pour l'exploitation des océans, Centre océanologique de Bretagne (CNEXO/COB), 49 p.

ONEDD, JICA -Observatoire National de l'Environnement et du Développement Durable, Agence Japonaise de Coopération International- (2007). Etat d'avancement Projet de coopération technique ONEDD-JICA renforcement de capacité de la surveillance environnementale en Algérie. Compte rendu des ateliers et séminaires conjoints Algérie Japon sur les normes de qualité et les efforts de protection de l'environnement 2007, Alger, pp 72-86.

RAMADE F. (1991). Conservatoire des écosystèmes méditerranéens (enjeux et perspectives). Les Fascicules du Plan Bleu III. Economica, Paris.

ROBBE D. (1981). Pollution métalliques du milieu naturel. Guide méthodologiques de leur étude à partir des sédiments. Rapport Bibliographique. Rapport de recherche LPC 104. Ministère de l’Urbanisme et du Logement, Ministère des Transports, Paris, 83 p.

SCHWARZENBACH R.P., GSCHWEND P.M., IMBODEN D.M. (2003). Environmental Organic Chemistry. Wiley-Interscience, NJ. 